\title{
Establishment of the Embryonic Shoot Meristem Involves Activation of Two Classes of Genes with Opposing Functions for Meristem Activities
}

\author{
Mitsuhiro Aida 1,*(D), Yuka Tsubakimoto ${ }^{2}$, Satoko Shimizu ${ }^{2}$, Hiroyuki Ogisu ${ }^{2}$, \\ Masako Kamiya $^{2}$, Ryosuke Iwamoto ${ }^{2}$, Seiji Takeda 2,3 ${ }^{\mathbb{D}}$, Md Rezaul Karim ${ }^{2,4}$, \\ Masaharu Mizutani ${ }^{5}$, Michael Lenhard ${ }^{6}$ and Masao Tasaka ${ }^{2}$ \\ 1 International Research Organization for Advanced Science and Technology (IROAST), \\ Kumamoto University, 2-39-1 Kurokami, Chuo-ku, Kumamoto 860-8555, Japan \\ 2 Graduate School of Biological Sciences, Nara Institute of Science and Technology, 8916-5 Takayama, \\ Nara 630-0192, Japan; moritsubaki.panda@gmail.com (Y.T.); s-s.combination@docomo.ne.jp (S.S.); \\ h.ogisu@icloud.com (H.O.); masadecosmos@gmail.com (M.K.); r.ftvm22@ymobile.ne.jp (R.I.); \\ seijitakeda@kpu.ac.jp (S.T.); mrkarim1996@yahoo.com (M.R.K.); m-tasaka@bs.naist.jp (M.T.) \\ 3 Graduate School of Life and Environmental Sciences, Kyoto Prefectural University, Hangi-cho 1-5, \\ Shimogamo, Sakyo-ku, Kyoto 606-8522, Japan \\ 4 Department of Horticulture, Bangladesh Agricultural University, Mymensingh 2202, Bangladesh \\ 5 Graduate School of Agricultural Science, Kobe University, 1-1 Rokkodai, Nada-ku, Kobe 657-8501, Japan; \\ mizutani@gold.kobe-u.ac.jp \\ 6 University of Potsdam, Institute for Biochemistry and Biology, Karl-Liebknecht-Str. 25-26, \\ 14476 Potsdam-Golm, Germany; michael.lenhard@uni-potsdam.de \\ * Correspondence: m-aida@kumamoto-u.ac.jp
}

Received: 31 July 2020; Accepted: 12 August 2020; Published: 15 August 2020

check for updates

\begin{abstract}
The shoot meristem, a stem-cell-containing tissue initiated during plant embryogenesis, is responsible for continuous shoot organ production in postembryonic development. Although key regulatory factors including KNOX genes are responsible for stem cell maintenance in the shoot meristem, how the onset of such factors is regulated during embryogenesis is elusive. Here, we present evidence that the two KNOX genes STM and KNAT6 together with the two other regulatory genes BLR and LAS are functionally important downstream genes of CUC1 and CUC2, which are a redundant pair of genes that specify the embryonic shoot organ boundary. Combined expression of STM with any of KNAT6, BLR, and LAS can efficiently rescue the defects of shoot meristem formation and/or separation of cotyledons in $c$ c 1 cuc2 double mutants. In addition, CUC1 and CUC2 are also required for the activation of $K L U$, a cytochrome P450-encoding gene known to restrict organ production, and KLU counteracts STM in the promotion of meristem activity, providing a possible balancing mechanism for shoot meristem maintenance. Together, these results establish the roles for CUC1 and CUC2 in coordinating the activation of two classes of genes with opposite effects on shoot meristem activity.
\end{abstract}

Keywords: shoot meristem; embryogenesis; stem cell; boundary; transcription factor; cytochrome P450; CUC; STM; LAS; BLR; KNAT6; KLU; CYP78A5

\section{Introduction}

Primary growth in plant shoots depends on the activity of stem-cell-containing tissue called the shoot meristem, which is located at the tip of the stem [1]. The shoot meristem is initially formed during embryogenesis and is activated upon germination to produce shoot organs such as leaves, 
stems, and floral organs, while it maintains an undifferentiated stem cell population at its center. Once activated, the shoot meristem keeps the balance between cell proliferation and differentiation to maintain an appropriate size of the stem cell population within it; factors essential for this process have been identified [2,3]. Although activation of these maintenance factors is associated with shoot meristem initiation during embryogenesis, how the process is coordinated is unknown.

Several key regulators for shoot meristem initiation have been reported [4-11]. Among them, the NAM/CUC3 type of NAC-domain transcription factors represents a class of regulators that are required for specification of shoot organ boundaries, which are sites for shoot meristem formation in embryonic and postembryonic development [12-16]. In Arabidopsis thaliana, the three NAM/CUC3 genes CUC1, CUC2, and CUC3 are expressed in cells along the boundary between two cotyledon primordia and promote shoot meristem formation and the separation of cotyledons $[5,6,17]$. As shoot meristem formation proceeds, expression of these genes is downregulated from the meristem center and becomes restricted to the adaxial and lateral boundaries of cotyledons. In postembryonic development, the three CUC genes are expressed at the adaxial and lateral boundaries of leaf primordia and are required for the formation of axillary shoot meristem as well as for the separation of leaves $[6,18]$.

Several genes whose expression is dependent on CUC gene activities have been identified. Expression of the two KNOTTED1-like homeobox (KNOX) genes SHOOT MERISTEMLESS (STM) and KNOTTED1-like from Arabidopsis thaliana 6 (KNAT6), which are required for shoot meristem maintenance, is absent from the $c u c 1$ cuc2 double mutant $[17,19]$ and ectopic expression of the CUC genes induces STM expression $[5,20,21]$. The LIGHT-DEPENDENT SHORT HYPOCOTYLS (LSH) genes LSH3 and LSH4, which encode nuclear proteins of the Arabidopsis LSH1 and Oryza G1 (ALOG) family, have been identified as direct transcriptional targets of the CUC1 protein and their overexpression induces ectopic shoot meristem formation [22]. Genome-wide mapping of protein-DNA interactions among boundary-enriched genes has identified the GRAS family gene LATERAL SUPPRESSOR (LAS) and the microRNA gene MIR164C as direct transcriptional targets of CUC2 [23]. LAS encodes a putative transcriptional regulator and is required for axillary shoot meristem formation [24]. Together, these analyses indicate that CUC genes regulate multiple genes involved in shoot meristem activity or boundary specification, or both. However, the functional relationship between these downstream genes and CUC gene activity remains elusive.

Here, we selected a set of genes whose expression is dependent on CUC1 and CUC2 during embryogenesis and demonstrated that the combined activities of STM, KNAT6, BLR, and LAS are important for promoting shoot meristem formation and cotyledon separation downstream of CUC1 and CUC2. Moreover, CUC1 and CUC2 are also required for the expression of KLUH $(K L U) / C Y P 78 A 5$, a cytochrome P450-encoding gene involved in the rate of shoot organ production and organ size $[25,26]$. Genetic analysis indicates that $K L U$ restricts shoot meristem activity and counteracts STM function. Our results thus indicate that the activation of two classes of genes with opposing functions, one positively and the other negatively affecting meristem activity, is an important step for shoot meristem formation.

\section{Results}

\subsection{Selection of Candidate CUC1 and CUC2 Downstream Genes}

It has been reported that the two KNOX genes STM and KNAT6, the GRAS gene LAS, and the two ALOG genes LSH3 and LSH4 show overlapping expression patterns to those of CUC1 and CUC2 in the boundary region of cotyledons, and their expression is absent in the corresponding region of cuc1 cuc2 double-mutant embryos [5,6,17,19,22]. We identified six additional candidate downstream genes positively regulated by CUC1 and/or CUC2 from microarray-based screening combined with quantitative real-time polymerase chain reaction (qRT-PCR) and in situ hybridization experiments (Supplementary Text S1; Supplementary Tables S1-S3). These genes were expressed in the boundary region that overlapped with the CUC gene expression domain $[5,17]$ and were downregulated 
specifically in the corresponding region of cuc1 cuc2 embryos (Figure 1A-D; Supplementary Figure S1), indicating the dependence of their expression on CUC1 and CUC2 activities during embryogenesis.
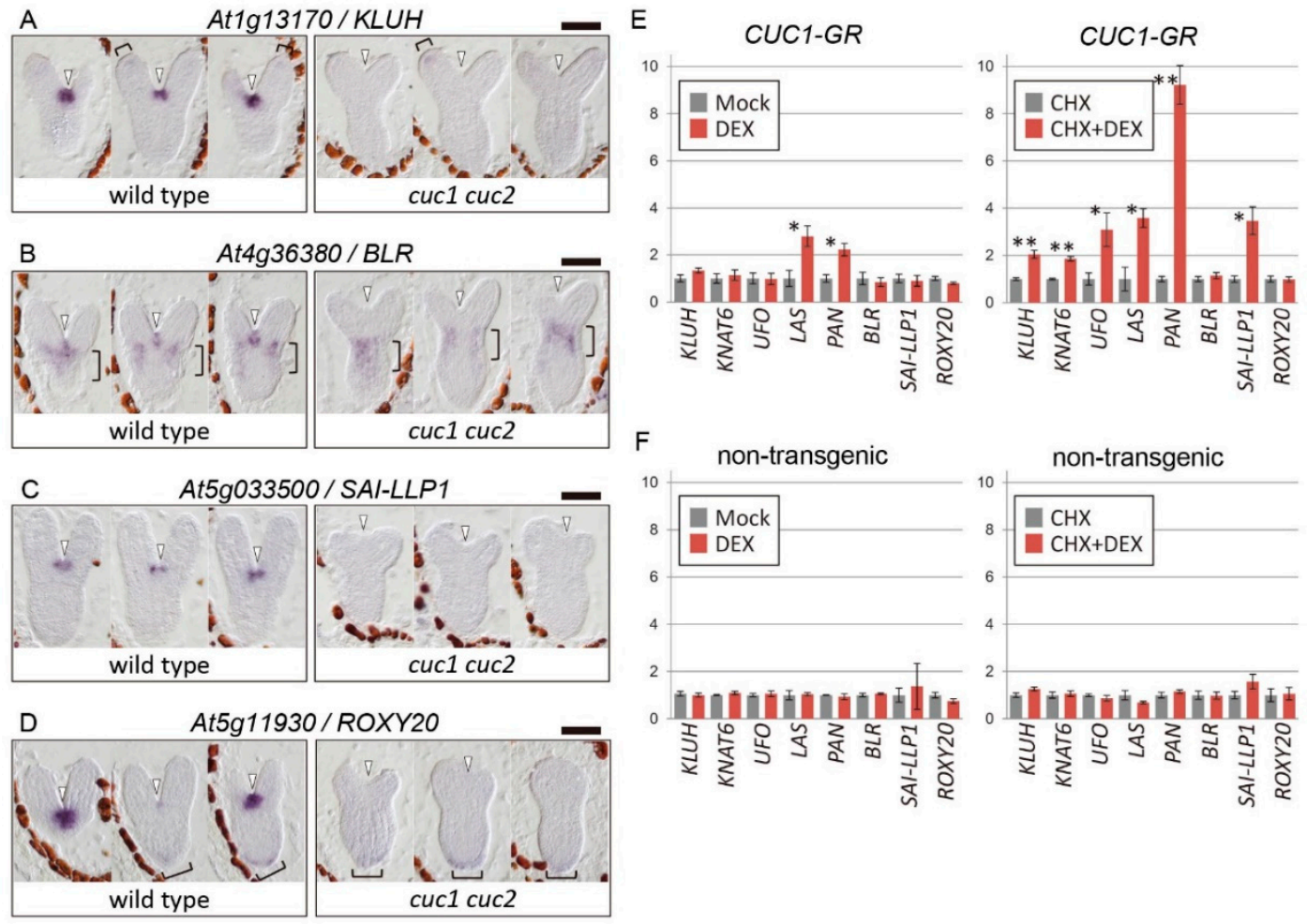

Figure 1. Regulation of candidate downstream genes by CUC1 and CUC2. (A-D) In situ hybridization of newly identified candidate downstream genes. Four of the six candidates are shown. Three serial longitudinal sections of wild-type Ler (left) and cuc1-1 cuc2-1 double-mutant (right) embryos at the late heart stage. Arrowheads indicate the position of the cotyledon boundary region. Brackets in (A), (B), and (D) indicate the position of expression outside the boundary region. Bars $=50 \mu \mathrm{m}$. (E,F) Transcriptional responses of candidate downstream genes upon dexamethasone (DEX) treatment in CUC1-GR (E) and non-transgenic (F) plants in the absence (left) or presence (right) of the protein synthesis inhibitor cycloheximide (CHX). Three biological replicates of 7-day-old seedlings. Single and double asterisks indicate $p<0.05$ and $p<0.01$, respectively, in comparisons between samples with and without DEX (Welch's $t$-test).

To gain insight into how CUC1 regulates expression of these candidate genes, we used the glucocorticoid receptor (GR) system, in which the activity of CUC1 is induced by the exogenous application of dexamethasone (DEX) [27]. Using this system, we previously found evidence for the direct activation of the LSH3, LSH4, and STM genes by CUC1 [22,28]. Among the remaining eight genes, we found that only $L A S$ and PAN were significantly upregulated upon DEX treatment alone in CUC1-GR plants (Figure 1E, left panel). By contrast, treating with both DEX and the protein synthesis inhibitor cycloheximide (CHX), which blocks secondary transcriptional responses caused by genes directly activated by CUC1, significantly upregulated not only $L A S$ and PAN, but also four additional genes among the eight tested. Treatment with $\mathrm{CHX}$ alone did not alter their expression levels (Figure 1E, right panel). Together, the results suggest that the six genes are under direct transcriptional regulation by the CUC1-GR protein, but that the action of CUC1 is counteracted by a CHX-sensitive negative factor with respect to activation of four of the genes (KLUH, KNAT6, UFO, and SAI-LLP1). Another possibility is that DEX treatment alone indirectly promotes expression of genes that negatively affect CUC1-dependant activation of the four genes. In control non-transgenic plants, which do not express CUC1-GR, none of the genes were upregulated by DEX treatment in the presence or absence of 
CHX (Figure 1F), indicating that the induction of the downstream genes was not a secondary effect of DEX or CHX.

\subsection{Combined Expression of STM with LAS, BLR, and KNAT6 is Sufficient to Rescue the Embryonic Shoot Phenotypes of cuc1 cuc2}

To examine the functional significance of the candidate genes in processes downstream of CUC1 and CUC2, we expressed each gene in the cuc1 cuc2 double-mutant background under the control of the CUC2 promoter (ProCUC2), which drives expression in the boundary region in both wild-type and double-mutant embryos (Figure 2A,B). In wild type, seedlings develop a shoot that continuously produces leaves immediately after germination and have two completely separated cotyledons (Figure 2C). By contrast, the cuc1 cuc2 double mutant has two cotyledons fused along their margins and fails to form a shoot, and this phenotype is fully penetrant (Figure 2D) [4]. When the coding sequence of CUC2 was used as a positive control (ProCUC2:CUC2), 41.7\% of the T1 seedlings showed a strongly rescued phenotype with no or slight delay in shoot formation and with completely separated cotyledons, $50.0 \%$ showed a mildly rescued phenotype with delayed or no shoot formation and half-separated cotyledons, and the remaining $8.3 \%$ showed non-rescued phenotype identical to that of cuc1 cuc2 (Table 1, Supplementary Figure S2A,B).

Among the 10 downstream genes, the ProCUC2:STM and ProCUC2:LAS transgenes were able to mildly rescue the $c u c 1$ cuc2 phenotype, resulting in the occasional formation of a functional shoot that can produce leaves as well as in the partial separation of cotyledons (Table 1, Figure 2E, and Supplementary Figure S2C-E). In cleared seedlings, wild type has the dome-shaped shoot meristem with a few leaf primordia, whereas cuc1 cuc2 plants lack either structure (Figure 2F,G) [4]. On the other hand, plants partially rescued by ProCUC2:STM showed variable phenotypes: some lacked a shoot meristem and leaf primordia, some developed small undifferentiated tissue, and the other produced the shoot meristem and leaf primordia (Figure $2 \mathrm{H}$ ). These results indicate that STM and LAS play prominent roles in shoot meristem formation and cotyledon separation and that their individual activities can partially bypass the requirements for CUC1 and CUC2 for embryonic shoot meristem formation and cotyledon separation.

The rescue of the $c u c 1$ cuc2 mutant phenotype by the STM or LAS transgene alone was only mild and partial, thus we next tested their combined activities. In the $F_{2}$ generation of the cross between the lines with the STM and LAS transgenes, only plants carrying both showed a rescued phenotype (Table 2). These rescued plants showed either partial (Figure 2J) or complete separation of cotyledons (Figure $2 \mathrm{~K}$ ), with the latter forming leaves with only a slight delay compared with the timing in the wild type, indicating that combined expression of STM and LAS is sufficient to compensate for the loss of CUC1 and CUC2 activities.

We next selected five other genes encoding transcription factors or transcriptional co-regulators, and tested their ability to rescue the $c u c 1$ cuc2 phenotype in combination with the STM transgene (Table 3). BLR and KNAT6 were able to achieve rescue when combined with STM (Figure 2K,L), while the rest failed to do so. Plants expressing both $S T M$ and $B L R$ produced nearly normal shoots with completely separated cotyledons (Figure $2 \mathrm{~K}$ ), indicating that, similarly to $L A S, B L R$ can efficiently support the ability of STM to promote shoot meristem formation and cotyledon separation in the absence of CUC1 and CUC2. By contrast, plants expressing both STM and KNAT6 only rescued the cotyledon phenotype, but not that of shoot formation (Figure 2L), indicating that KNAT6 can support the STM activity only in the limited developmental pathway downstream of the CUC genes. 
A

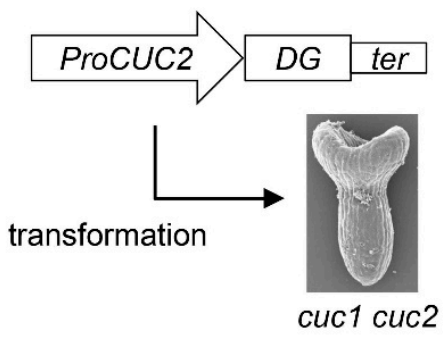

B
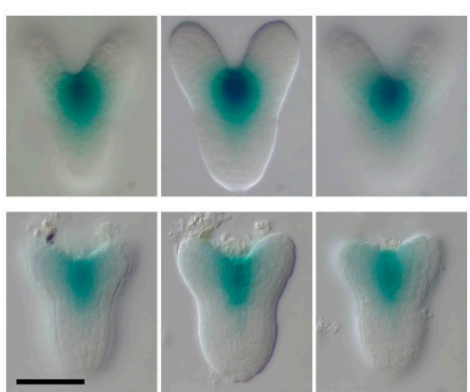

-
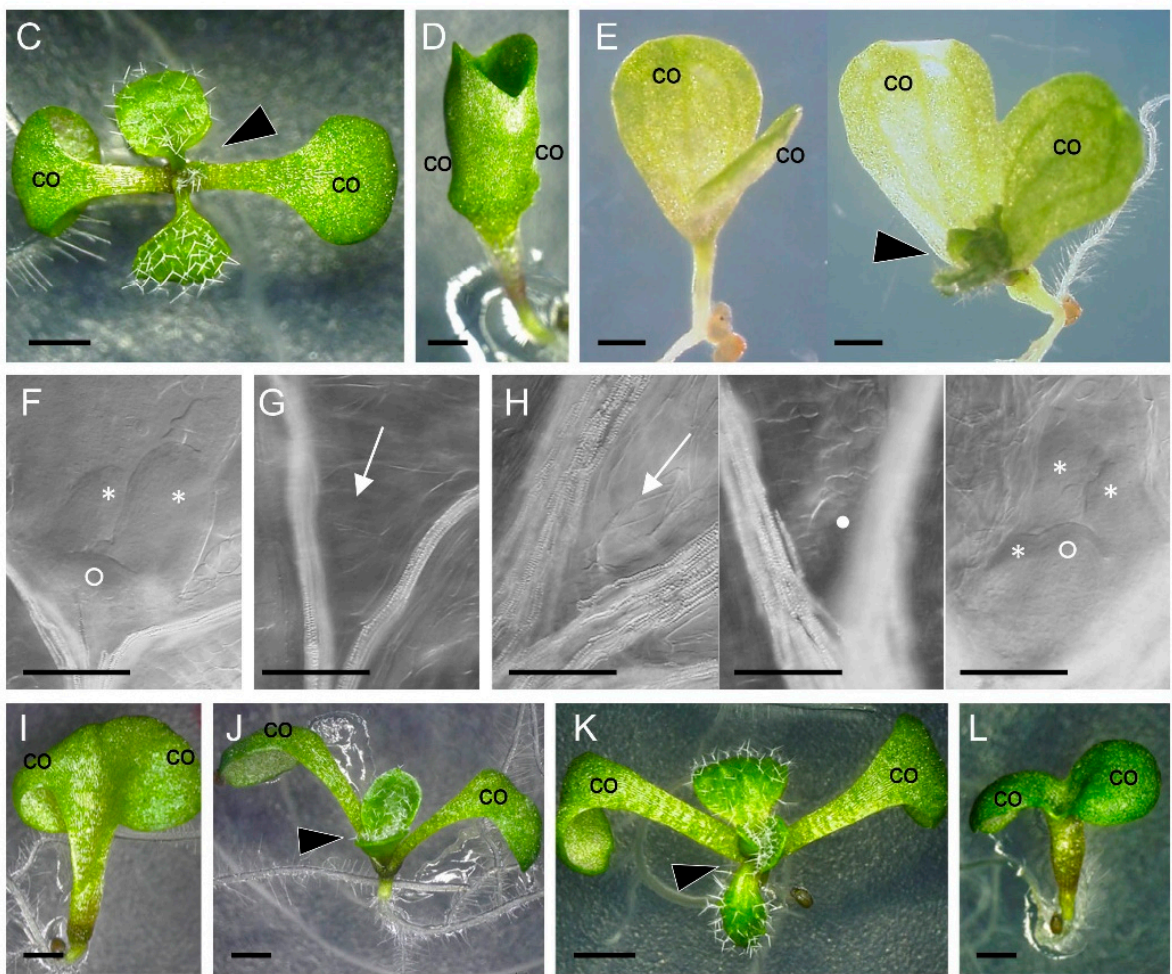

Figure 2. Rescue of $c u c 1$ cuc2 phenotype by candidate downstream genes. (A) Schematic diagram of rescue experiments. CUC2 promoter (ProCUC2), cDNA of downstream gene (DG), and nos terminator (ter). (B) CUC2 promoter activity detected by $\beta$-glucuronidase (GUS). Longitudinal views of GUS-stained wild-type Col (top) and cuc1-5 cuc2-3 (bottom) embryos in three different optical sections, showing expression in the boundary region. (C,D) Seedlings of wild-type Col (C) and cuc1-5 cuc2-3 (D), 7 days after germination (dag). Wild type has two separated cotyledons (co) and develops a shoot between them (arrowhead), whereas cuc1-5 cuc2-3 has cotyledons (co) fused along their margins. (E) A cuc1-5 cuc2-3 seedling mildly rescued by the STM transgene at 9 dag (left) and 16 dag (right). Cotyledons (co) are partially fused on one side. Note that the shoot is only visible at 16 dag (arrowhead). (F,G) Shoot apices in cleared seedlings (11 dag) of wild type (F) and cuc1-5 cuc2-3 (G). Wild type develops the shoot meristem (open circle) and leaf primordia (asterisks), whereas cuc1-5 cuc2-3 lacks these structures at the corresponding postion (arrow). (H) Shoot apices in cleared seedlings (11 dag) of cuc1- 5 cuc2-3 rescued by STM, showing variable phenotypes: no visible shoot meristem and leaf primordia (left, arrow), small undifferentiated tissue (middle, closed circle), and shoot meristem with leaf primordia (right, open circle, and asterisks). (I-L) cuc1-5 cuc2-3 seedlings (9 dag) rescued by combined transgenes: STM and LAS (I,J), STM and BLR (K), and STM and KNAT6 (L). Arrowheads represent emerging shoot. co, cotyledon. Bars $=50 \mu \mathrm{m}(\mathbf{B}, \mathbf{F}-\mathbf{H}) ; 1 \mathrm{~mm}(\mathbf{C}-\mathbf{E}, \mathbf{I}-\mathbf{L})$. 
Table 1. Rescue of $c u c 1$ cuc2 seedling phenotype by downstream gene expression under the control of CUC2 regulatory sequence.

\begin{tabular}{ccccc}
\hline Transgene & No Rescue $^{\mathbf{a}} \mathbf{( \% )}$ & Mild Rescue $^{\mathbf{b}} \mathbf{( \% )}$ & Strong Rescue $^{\mathbf{c}} \mathbf{( \% )}$ & Total Number of T1 Seedlings $^{\text {(\%) }}$ \\
\hline KLU & 100 & 0 & 0 & 25 \\
KNAT6 & 100 & 0 & 0 & 15 \\
UFO & 100 & 0 & 0 & $20^{\mathrm{d}}$ \\
LAS & 86.5 & 13.5 & 0 & 11 \\
STM & 54.5 & 45.5 & 0 & 12 \\
PAN & 100 & 0 & 0 & 31 \\
LSH4 & 100 & 0 & 0 & 15 \\
BLR & 100 & 0 & 0 & 12 \\
SAI-LLP1 & 100 & 0 & 0 & 12 \\
ROXY20 & 100 & 0 & 41.7 & 12 \\
CUC2 & 8.3 & 50.0 & 0 & 4 \\
GUS & 100 & 0 & 0
\end{tabular}

${ }^{a}$ Cotyledons were fused along both sides with only a small split at their tips and no shoot was formed.

$\mathrm{b}$ Cotyledons were fused along one or both sides with more than half of their margins split. No shoot was formed or a shoot became visible only after 9 dag. ${ }^{c}$ Cotyledons were completely separated and a shoot was visible by 9 dag. ${ }^{\mathrm{d}}$ One seedling had flat and round green tissue on top of the hypocotyl instead of a cup-shaped cotyledon. This is classified as "No rescue", as neither shoot formation nor cotyledon separation occurred.

Table 2. Effect of combined expression of STM and LAS on the phenotype of $c u c 1$ cuc2.

\begin{tabular}{|c|c|c|c|c|c|c|}
\hline Transgene A & Transgene B & No Rescue ${ }^{a}(\%)$ & Mild Rescue $^{\text {b }}(\%)$ & $\begin{array}{c}\text { Strong } \\
\text { Rescue }^{\mathrm{c}}(\%)\end{array}$ & $\begin{array}{c}\text { Total Number of } \\
\text { F2 Seedlings }\end{array}$ & Group $^{d}$ \\
\hline STM & - & 100 & 0 & 0 & 11 & a \\
\hline- & LAS & 100 & 0 & 0 & 26 & $\mathrm{a}$ \\
\hline STM & LAS & 0 & 71.1 & 28.9 & 38 & $b$ \\
\hline
\end{tabular}

${ }^{a}$ Cotyledons were fused along both sides with only a small split at their tips and no shoot was formed.

${ }^{b}$ Cotyledons were fused along one or both sides with more than half of their margins split. No shoot was formed or a shoot became visible only after 9 dag. ${ }^{c}$ Cotyledons were completely separated and a shoot was visible by 9 dag. ${ }^{\mathrm{d}}$ Different letters indicate statistically significant differences $(p<0.01$, Fisher's exact test with Holm multiple testing correction).

Table 3. Effect of combined expression of STM and other downstream genes on the phenotype of cuc1 cuc2.

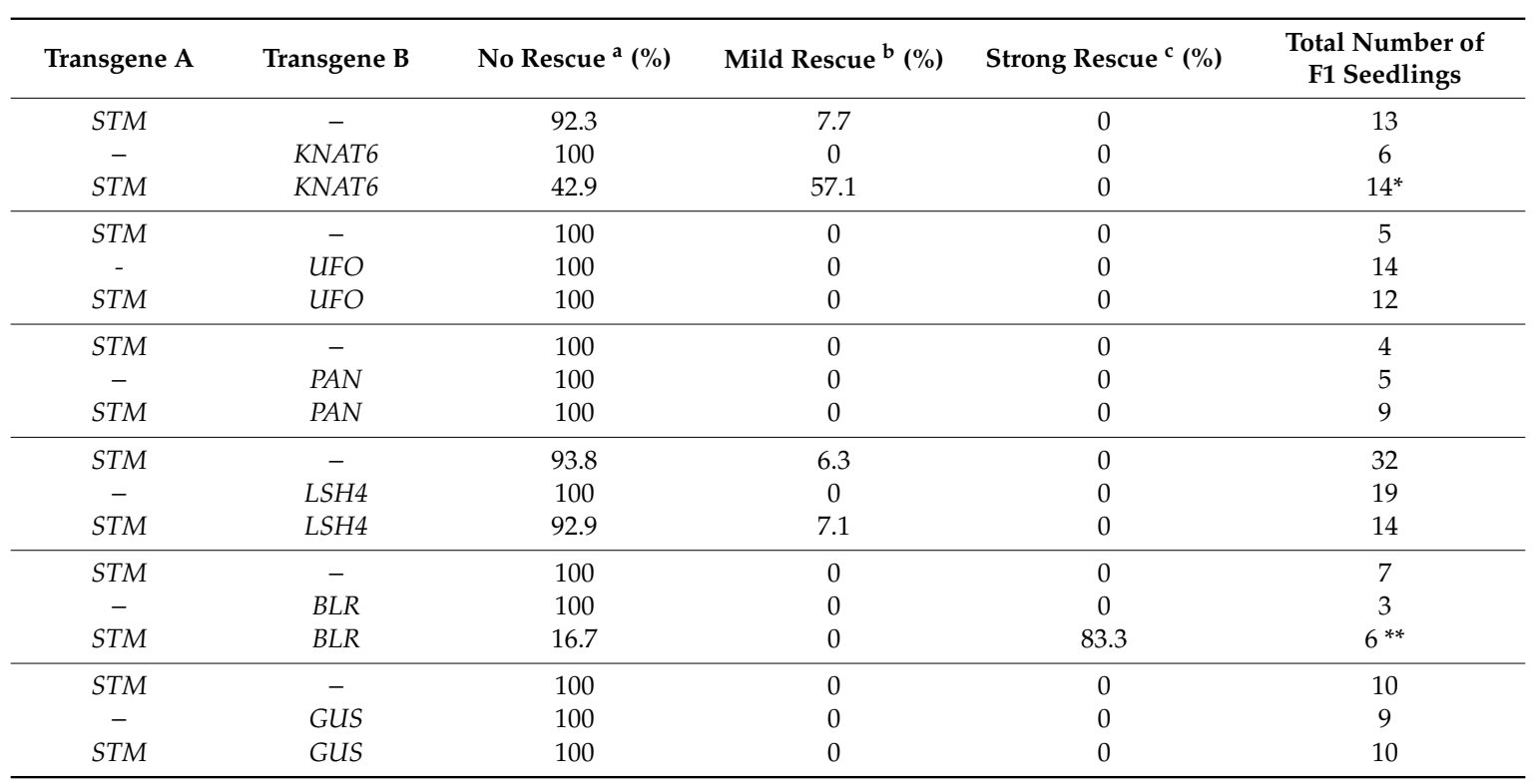

a Cotyledons were fused along both sides with only a small split at their tips and no shoot was formed. $\mathrm{b}$ Cotyledons were fused along one or both sides with more than half of their margins split. No shoot was formed or a shoot became visible only after 9 dag. ${ }^{c}$ Cotyledons were completely separated and a shoot became visible by 9 dag. Asterisks indicate significant differences in the ratio of the phenotypes of plants carrying the transgene B alone and those carrying both transgenes A and B $\left({ }^{*} p<0.05,{ }^{* *} p<0.01\right.$; Fisher's exact test). 
2.3. Combined Loss of Function of STM, LAS, BLR, and KNAT6 Severely Impairs Shoot Meristem Formation and Cotyledon Separation

We then tested the combined effect of the loss-of-function mutations in the four genes (STM, LAS, $B L R$, and KNAT6) in young seedlings. Among the single mutants of these genes, only stm shows defects in shoot development with complete penetrance. In the case of the strong allele stm-1C [29], the mutant typically stops leaf formation after producing the first two leaves (Figure 3A-D). It has been reported that mutations in BLR or KNAT6 alone do not cause visible phenotypes in seedlings, but enhance the defects of $s t m$ mutants $[19,30]$. Consistent with this, we found that the stm-1C blr double mutant produced fewer leaves than stm-1C (Figure 3A,C,D) and showed delayed first-leaf growth (Figure 3C,E). In addition, stm-1C blr showed a higher frequency of cotyledon fusion than stm-1C (Table 4). The stm-1C knat6 double mutant showed strong cotyledon fusion and the lack of leaf production and both phenotypes were observed with complete penetrance (Figure 3F). These results confirmed the previous results that BLR and KNAT6 are required for shoot meristem formation and cotyledon separation.

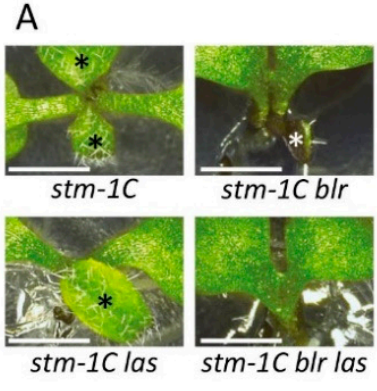

B

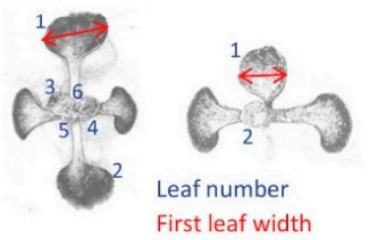

D
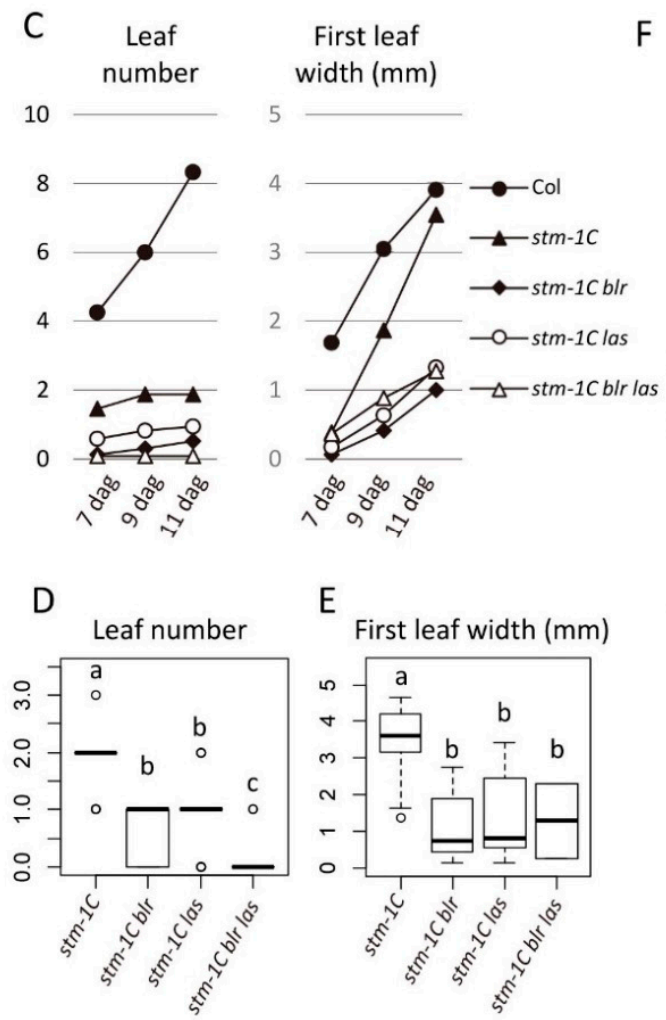

E

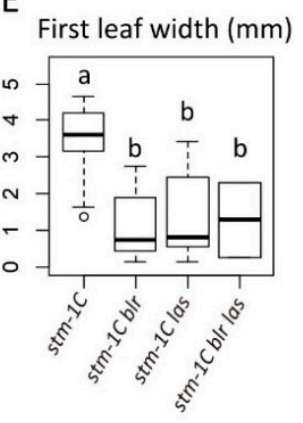

$\mathrm{F}$

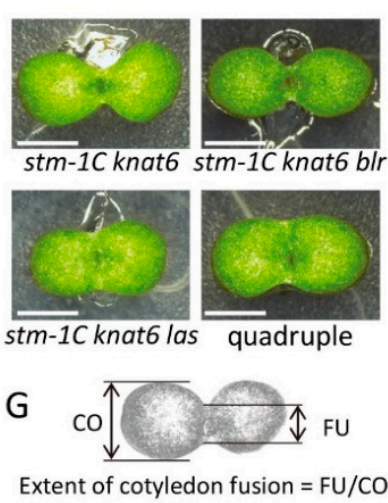

$\mathrm{H}$

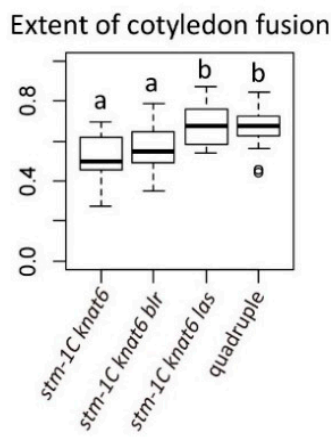

Figure 3. Genetic interactions of $s t m$, las, blr, and knat6 mutants. (A) The las mutation enhances the shoot production phenotype of $s t m-1 C$ and stm-1C blr mutants. Shoot apex at 9 dag. Asterisks indicate developing leaves. (B) Examples of shoot phenotype measurements in the wild-type $\mathrm{Col}$ (left) and stm-1C (right). (C) Change in leaf number (left) and first leaf width (right). (D,E) Box plots of leaf number (D) and first leaf width (E) at 11 dag. (F) las enhances the cotyledon fusion phenotype of stm-1C knat6 and stm-1C knat6 blr mutants. Seedlings at 7 dag. (G) Quantification of cotyledon fusion. (H) Box plot showing extent of cotyledon fusion at 7 dag in each genotype. Different letters in box plots indicate statistically significant differences $(p<0.01$, Steel-Dwass method for (D); $p<0.05$, Tukey-Kramer method for (E) and (H)). Sample size is 24, 23, 17, and 23 for (C) (left) and (D); 24, 12, 13, and 2 for (C) (right) and (E); and 11, 23, 12, and 19 for (H). Bars in (A) and (E), $2 \mathrm{~mm}$. 
Table 4. Frequency of cotyledon fusion in stm mutant combined with blr and las mutants.

\begin{tabular}{cccc}
\hline Genotype & Frequency of Fusion (\%) & Total & Group * \\
\hline Col & 0 & 55 & $\mathrm{a}$ \\
stm & 12.5 & 24 & $\mathrm{~b}$ \\
blr stm & 91.3 & 23 & $\mathrm{c}$ \\
las stm & 35.3 & 17 & $\mathrm{~b}$ \\
bl las stm & 95.7 & 23 & $\mathrm{c}$
\end{tabular}

* Different letters indicate statistically significant differences $(p<0.01$, Fisher's exact test with Holm multiple testing correction).

Similar to blr and knat6 single mutants, young seedlings of the las single mutant reportedly show a normal appearance, except for a very small proportion of plants with fused cotyledons $(0.26 \%)$ [6]. However, we found that the las mutation enhanced defects in shoot meristem activity when combined with the stm-1C single or stm-1C blr double mutant (Figure 3A-E). In addition, the las mutation enhanced the cotyledon fusion phenotype of both stm-1C knat6 and stm-1C blr knat6 mutants (Figure 3F-H). These results show that the $L A S$ gene contributes to embryonic shoot meristem formation and cotyledon separation independently of STM, KNAT6, and BLR. The blr knat6 las triple mutant was phenotypically normal. Together, our results demonstrate that the four transcription factor-encoding genes STM, $K N A T 6, B L R$, and LAS play key roles for embryonic shoot meristem formation and cotyledon separation downstream of CUC1 and CUC2.

\subsection{The KLUH Gene Restricts the Embryonic Shoot Meristem and Counteracts STM}

Among the downstream target genes, KLU/CYP78A5 encoding a cytochrome P450 enzyme of the CYP78A family plays postembryonic roles in shoot organ size and organ production rate [25,26], raising the possibility that this gene also affects shoot meristem activity. Moreover, the mutation in rice PLA1, a member of the same family, causes an enlarged shoot meristem phenotype [31,32]. Indeed, we found that the two independent $k l u$ insertion alleles $(k l u-019348$ and $k l u-4)$ were associated with precocious leaf initiation in young seedlings (Figure 4A-F), suggesting enhanced shoot meristem activity. In addition, the width of the shoot meristem was significantly greater in $k l u$ than in the wild type (Figure 4G). Furthermore, embryos of the klu mutants displayed an enlarged cotyledon boundary region (Figure $4 \mathrm{H}-\mathrm{J}$ ) and this phenotype was associated with an enlarged expression domain of the shoot stem cell marker CLV3::GUS [33] (Figure 4K,L). These results indicate that the $K L U$ gene is required for restricting shoot meristem size and activity during embryogenesis and postembryonic development.

The identification of KLU as a downstream gene of CUC1 and CUC2 was unexpected because $K L U$ negatively affects shoot meristem activity, whereas the CUC genes are positive regulators of shoot meristem formation. To further investigate the relationship between KLU and the CUC genes, we crossed the $k l u$ mutant with the $c u c 1$ cuc2 double mutant and examined their genetic interactions. In single mutants of $k l u, c u c 1$, and $c u c 2$, all seedlings produced a functional shoot as the wild type, and except for a small fraction of $c u c 1$ mutants with partially fused cotyledons, their cotyledons were completely separated (Table 5; Figure 5A). Similarly, seedlings of klu cuc1 and klu cuc2 double mutants all produced a fully functional shoot and most of them developed completely separated cotyledons, whereas small fractions had partially fused cotyledons (Table 5; Figure 5B). Importantly, the klu cuc1 cuc2 triple mutant was indistinguishable from $c u c 1 c u c 2$ double mutants in that they formed strongly fused cup-shaped cotyledons and lacked a shoot meristem (Table 5; Figure 2D,G and Figure 5C-E). These results show that the $c u c 1 c u c 2$ double mutations are epistatic to $k l u$ and indicate that the $K L U$ gene can affect shoot meristem activity only when the functional CUC1 and CUC2 genes are present. 
Col
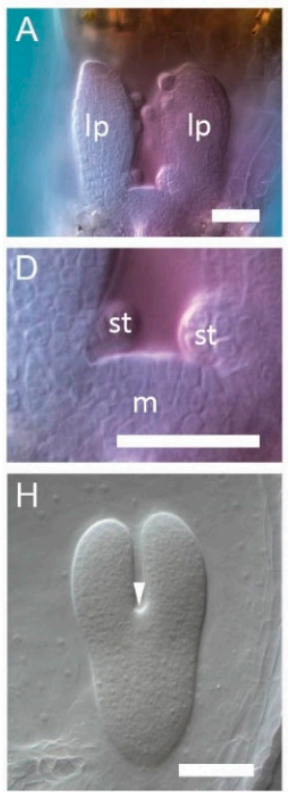

klu-019348
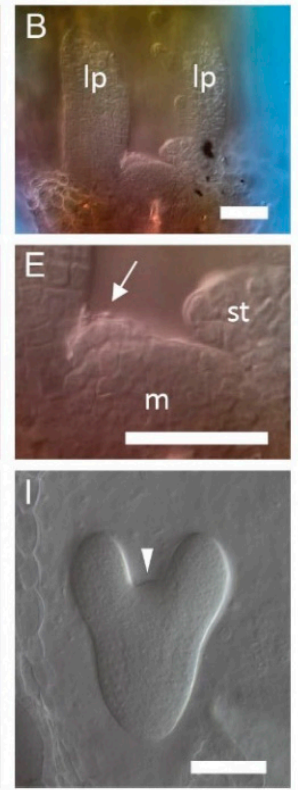

klu-4
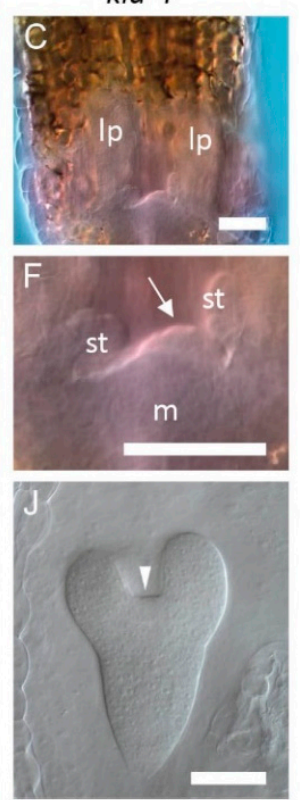

G

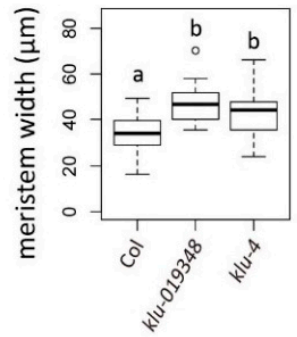

Col

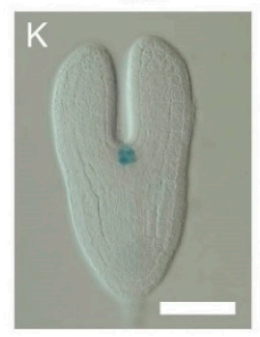

klu-4

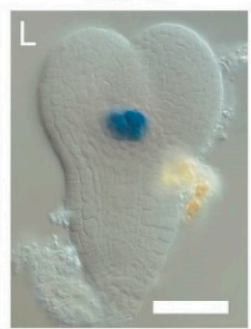

Figure 4. Mutations in $K L U$ cause shoot meristem enlargement and precocious leaf formation. (A-F) Shoot apex of wild type (A,D), klu-019348 (B,E), and $k l u-4$ (C,F) in cleared seedlings at 3 dag. (D-F) are close-up views of (A-C), respectively. Optical sections are photographed at a position slightly off-center to reveal the precociously formed third-leaf primordia in the $k l u$ mutants (arrows). lp, the first two leaf primordia; m, shoot meristem; st, stipule. (G) Shoot meristem width of wild type and two $k l u$ mutant alleles at $3 \mathrm{dag}$. Different letters in box plots indicate statistically significant differences ( $p<0.01$, Tukey-Kramer method). Sample size is 34, 28, and 42 for G. (H-J) Torpedo-stage embryos of wild type (H), klu-019348 (I), and klu-4 (J). Arrowheads indicate the position of the cotyledon boundary region. $(\mathbf{K}, \mathbf{L})$ CLV3::GUS expression in wild-type $(\mathbf{K})$ and $k l u-4(\mathbf{L})$ torpedo-stage embryos. Scale bar $=$ $50 \mu \mathrm{m}$.

Table 5. Genetic interactions among klu-4, cuc1-5, and cuc2-3.

\begin{tabular}{|c|c|c|c|c|}
\hline \multirow{2}{*}{ Genotype } & \multicolumn{3}{|c|}{ Phenotype } & \multirow{2}{*}{$\begin{array}{l}\text { Total Number } \\
\text { of Seedlings }\end{array}$} \\
\hline & Normal a (\%) & Weak $^{b}(\%)$ & Strong $^{\mathrm{c}}(\%)$ & \\
\hline Col & 100 & 0 & 0 & 76 \\
\hline$k l u$ & $100 *$ & 0 & 0 & 289 \\
\hline cuc1 & 95.6 & 4.4 & 0 & 135 \\
\hline сuс2 & 100 & 0 & 0 & 118 \\
\hline klu сис1 & 95.9 & 4.1 & 0 & 172 \\
\hline klu cuc2 & 97.2 & 2.8 & 0 & 217 \\
\hline сис1 сис2 & 0 & 0 & 100 & 50 \\
\hline klu сис1 сис2 & 0 & 0 & 100 & 54 \\
\hline
\end{tabular}

${ }^{a}$ Cotyledons were completely separated and a shoot was formed immediately after germination. ${ }^{\mathrm{b}}$ Cotyledons were partially fused and a shoot was formed immediately after germination. ${ }^{\mathrm{c}}$ Cotyledons were strongly fused, forming a cup shape, and no shoot was formed after two weeks of observation. * A very small fraction $(1.7 \%)$ had three cotyledons instead of two. 

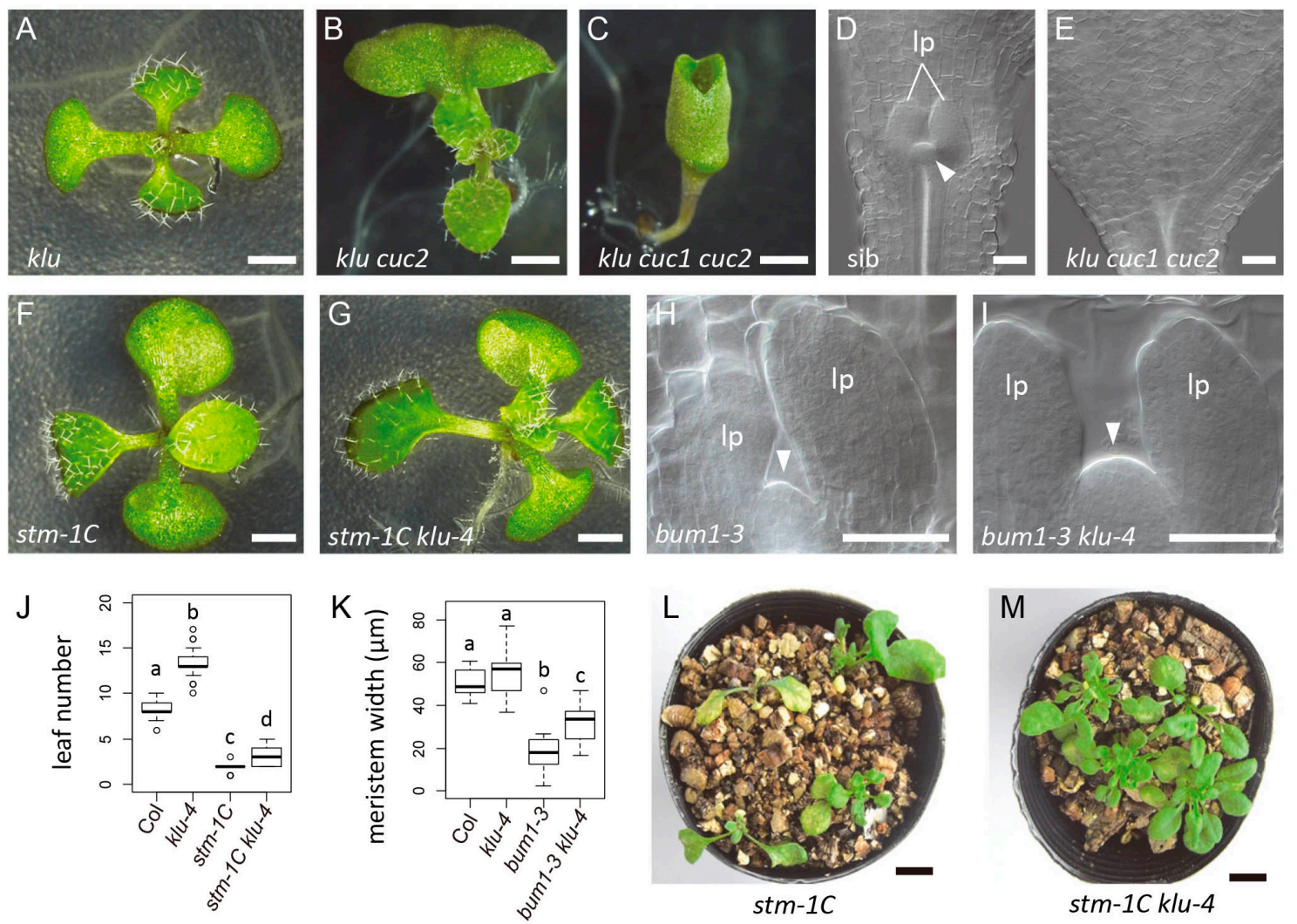

Figure 5. KLU acts downstream of CUC1 and CUC2, and counteracts STM in the regulation shoot meristem activity. (A-C) Seven-day-old seedlings of klu-4 (A), klu-4 cuc2-3 (B), and klu-4 cuc1-5 cuc2-3 (C). (D,E) Shoot apices of the progeny from $k l u-4 c u c 1-5$ cuc2-3/+ parent plants. A sib seedling with the shoot meristem (D) and a klu cuc1 cuc2 seedling without it (E). (F,G) Nine-day-old seedlings of the strong stm allele stm-1C (F) and stm-1C klu-4 (G). (H,I) Shoot apex of the weak stm allele bum1-3 (H) and bum1-3 klu-4 (I) at 4 dag. (J) Leaf number at 11 dag. (K) Shoot meristem width at 4 dag. $(\mathbf{L}, \mathbf{M})$ Twenty-nine-day-old plants of $s t m-1 C$ (L) and stm-1C klu-4 (M). Four plants are grown in each pot. Different letters in box plots indicate statistically significant differences $(p<0.01$, Steel-Dwass method for $(\mathbf{J}) ; p<0.05$, Tukey-Kramer method for $(\mathbf{K})$ ). Sample size is 55, 30, 24, and 14 for (J); 14, 18, 15, and 9 for $(\mathbf{K})$. Arrowheads indicate the shoot meristem. lp, leaf primordia. lp, leaf primordia. Scale bar, $1 \mathrm{~mm}$ for (A) to $(\mathbf{C}, \mathbf{F}, \mathbf{G}) ; 50 \mu \mathrm{m}$ for $(\mathbf{D}, \mathbf{E}, \mathbf{H}, \mathbf{I}) ; 10 \mathrm{~mm}$ for $(\mathbf{L})$ and (M).

Next, we examined the genetic interaction of $K L U$ with STM, which represents a functionally important class of CUC downstream genes that positively affects shoot meristem activity. Young seedlings of the stm $\mathrm{klu}$ double mutant produced more leaves and had an enlarged shoot meristem compared with stm single mutants (Figure 5F-K). Moreover, whereas the strong stm- $1 \mathrm{C}$ mutant allele typically arrested shoot growth after producing a few leaves (Figure 5L), the stm-1C klu-4 double mutant showed prolonged vegetative shoot growth (Figure 5M). Taken together, these results indicate that the KLU gene activity counteracts that of STM in postembryonic shoot development.

To clarify the mechanism by which $C U C 1$ and $C U C 2$ regulate KLU gene expression, we examined the expression of reporter genes containing cis-regulatory sequences of $K L U$. A reporter construct that carried regions $2 \mathrm{~kb}$ upstream and $0.6 \mathrm{~kb}$ downstream (Pro $2 \mathrm{~kb}$ ) showed activities in the cotyledon boundary region, cotyledon margins, and root pole (Figure 6A,B). This expression pattern resembled that of $K L U$ mRNA detected by in situ hybridization, except that the reporter activity in cotyledons was broader and that in the root pole was detected for a prolonged time (compare Figure 6B with Figure $1 \mathrm{~A}$ and Figure S1A), indicating that the Pro $2 \mathrm{~kb}$ reporter contained a set of cis-regulatory elements sufficient for driving the native expression pattern of the gene at least in the cotyledon boundary region. When this construct was introduced into the $c u c 1 c u c 2$ double-mutant background, its expression 
disappeared specifically in the cotyledon boundary region (Figure 6C), which corresponds to the region where the CUC genes act in normal development. Moreover, deletion of the region between 1 and $2 \mathrm{~kb}$ upstream of the gene resulted in the loss of expression in the cotyledon boundary region as well as in cotyledon margins (Figure 6A,D; Pro $1 \mathrm{~kb}$ ). These results indicate that this $1 \mathrm{~kb}$ region contains cis-regulatory elements required for CUC1- and CUC2-dependent transcription in the shoot apex.

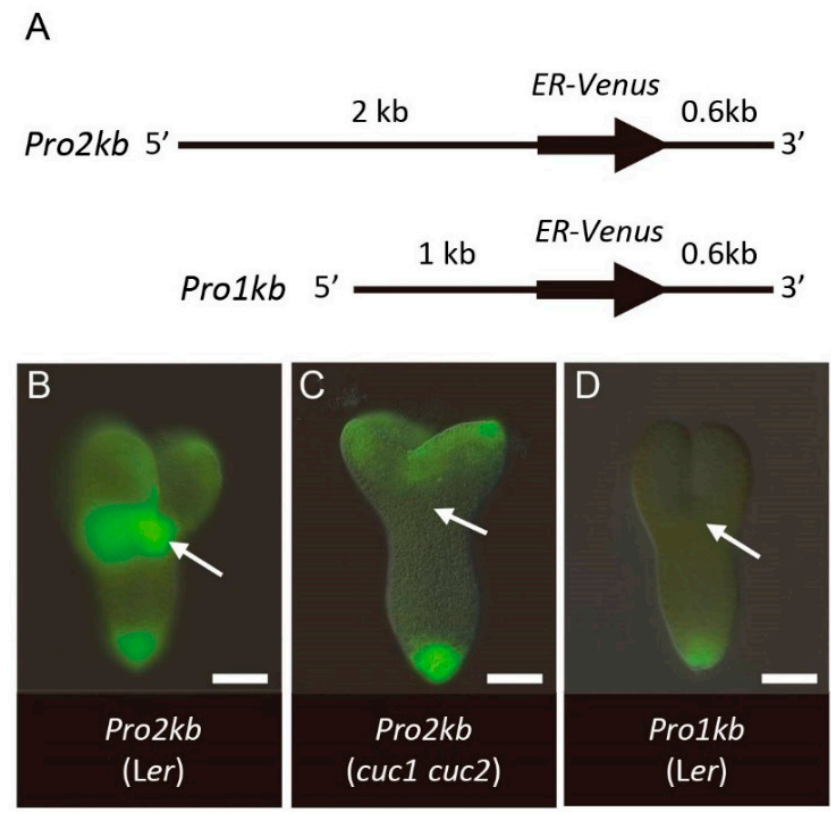

Figure 6. KLU expression is regulated by CUC1 and CUC2 via a specific promoter region. (A) Schematic diagram of the KLU reporter genes. (B-D) Expression of $K L U$ reporter genes in embryos. Pro2kb in wild type Ler (B), Pro2kb in cuc1-1 cuc2-1 (C), and Pro1kb in wild type Ler (D). Arrows indicate the position of the cotyledon boundary region of wild type (B,D) and the corresponding region of cuc1-1 cuc2-1 (C). Scale bar, $50 \mu \mathrm{m}$.

\section{Discussion}

In this work, through functional analyses of genes acting downstream of CUC1 and CUC2, we found that the combined activities of STM with LAS, BLR, and KNAT6 were important for shoot meristem formation and cotyledon separation. The abilities of these genes to rescue the $c u c 1 c u c 2$ mutant phenotypes when expressed under the boundary-specific promoter, together with the strong shoot meristem and cotyledon phenotypes observed in the quadruple mutant, support a model in which the CUC genes promote shoot meristem formation and cotyledon separation mainly through the activation of these four genes. It was previously shown that STM is a direct transcriptional target of CUC1 [28]. The experiments using the DEX-inducible CUC1-GR plants and CHX treatments in our current work indicate that LAS and KNAT6 are additional direct targets of the CUC1 protein, whereas the regulation of $B L R$ by CUC1 may be indirect.

Our analysis highlights the importance of the activation of STM expression by the CUC genes in shoot meristem formation and cotyledon separation. STM encodes a KNOX transcription factor [34] whose activity is continuously required for shoot meristem maintenance in postembryonic development through regulating various aspects of shoot meristem properties including pluripotency, self-maintenance, promotion of cell cycle, repression of differentiation, and hormone metabolism [28,35-37]. How STM promotes cotyledon separation is currently unknown, but its ability to repress growth and promote leaf dissection, when ectopically expressed, may be involved in the repression of growth at the cotyledon boundary [38].

The possible molecular mechanisms by which STM acts in concert with the rest of the four genes may vary. KNAT6 encodes a KNOX protein closely related to STM, so its ability to enhance the rescuing 
activity of STM can simply be explained by functional redundancy [19,39]. By contrast, BLR encodes a BEL-class homeodomain protein, which physically interacts with STM [30], and nuclear localization of STM requires its interaction with BLR [40-42]. These results indicate that the coexpression of $B L R$ with STM provides a sufficient amount of BLR-STM complex to the nucleus, thereby efficiently promoting shoot meristem formation and cotyledon separation. In axillary shoot meristem formation, another BEL protein, ATH1, which is functionally redundant with BLR, forms a heterodimer with STM and directly promotes the transcription of STM, thus forming a self-activation loop [43]. It is also possible that the formation of BLR-STM heterodimer during embryogenesis is critical for initiating the STM self-activation loop, allowing self-maintenance of the shoot meristem.

Our functional analyses also demonstrate that the LAS gene contributes to embryonic shoot meristem formation and cotyledon separation downstream of CUC1 and CUC2. LAS encodes a putative transcriptional regulator of the GRAS family and acts as a central hub in the gene regulatory network for axillary meristem formation downstream of CUC2 $[23,24]$. The precise mechanisms by which $L A S$ regulates these processes remain elusive; however, the mutation in the LAS ortholog in tomato affects the levels of hormones involved in shoot meristem activity, such as auxin, gibberellin (GA), and cytokinin $[44,45]$. Recently, it has been shown that the LAS protein binds to the promoter of GA20x4, which encodes a GA deactivation enzyme, and promotes its expression [46]. These results raise the possibility that $L A S$ reduces the levels of GA in the boundary region to promote shoot meristem activity, thereby contributing independently of STM, KNAT6, and BLR to the process downstream of the CUC genes.

In contrast to the above four genes, which are positive regulators of shoot meristem activity, $K L U / C Y P 78 A 5$ represents the functionally opposite class of downstream genes regulated by CUC1 and CUC2, as shown by the enhanced shoot meristem size and activity in the klu mutant as well as its genetic interaction with the stm mutant. Our data thus indicate that, by activating the two classes of genes with opposing functions during embryogenesis, the CUC genes create an optimal microenvironment for the shoot meristem to maintain its appropriate size and to produce organs at an appropriate rate. It has been well established that the balance between the self-renewal of stem cells and differentiation of their progeny is critical for postembryonic shoot meristem and that this balance is maintained by the WUS-CLV3 feedback system, which is supported by multiple transcription factors and plant hormones [3,47]. Our results provide an additional level of regulation for the balancing mechanism of shoot stem cell maintenance. Detailed functional analysis of the KLU gene as well as its relationship to previously known stem cell regulators will further improve our understanding of shoot meristem regulation.

\section{Materials and Methods}

\subsection{Plant Materials}

Arabidopsis thaliana accessions Columbia ( $\mathrm{Col})$ and Landsberg erecta $(\mathrm{Ler})$ were used as the wild type. CUC1-GR was established in the Ler background using the same construct as described previously [22]. CLV3::GUS was reported previously [33]. Expression analyses of genes downstream of CUC were carried out in cuc1-1 cuc2-1 [4,5]. For rescue experiments, cuc1-5 cuc2-3 was used [6]. Loss-of-function mutants of the downstream genes were as follows: klu-019348 (Sallk_019348) and klu-4 for KLU [25]; knat6-2 for KNAT6 [19]; las-101 for LAS [6]; stm-1C for STM [29]; and pny-40126 for BLR [48]. Details of the mutants are described in Supplementary Table S4.

\subsection{Constructs}

For ProCUC2:LAS, we amplified the LAS coding sequence derived from Col with the PCR primers BamHI-LAS-F (5'-TATCTGGATCCATGCTTACTTCCTTCAAATC- $3^{\prime}$ ) and EcoRI-LAS-R (5'-TTCTCGAATTCTCATTTCCACGACGAAACGG-3') and placed it upstream of the 355 terminator in a modified UAS cassette vector [49] using the EcoRI and BamHI sites. The BamHI-NotI fragment 
containing $L A S$ and the terminator was then placed downstream of the CUC2 promoter of $p B S-g C 2$, which contains a $5.9 \mathrm{~kb}$ fragment of the CUC2 genomic sequence [50], yielding ProCUC2:LAS BS. The SalI-NotI fragment of ProCUC2:LAS BS was then inserted into pBIN50, a modified binary vector carrying a kanamycin resistance gene [29]. To obtain ProCUC2:BLR, cDNA derived from Ler was amplified using the primers BLRcDNAfull-F (5'-TTTCCCATGGCTGATGCATA- $\left.3^{\prime}\right)$ and BLRcDNAfull-R (5' -TCAACCTACAAAATCATGTA-3'), cloned into pCR $^{\text {TM}}$-Blunt II-TOPO (Invitrogen, [Waltham, MA, USA]), and the resulting EcoRI fragment was then placed upstream of the $35 \mathrm{~S}$ terminator of the modified UAS cassette. Fusion with the CUC2 promoter and transfer to a binary vector was carried out in the same manner as that for ProCUC2:LAS, except that pBIN60, a modified binary vector with a sulfadiazine resistance gene, was used. To obtain the other chimeric constructs of the CUC2 promoter and downstream genes, the $3.1 \mathrm{~kb}$ SalI-BglII fragment of the CUC2 promoter was blunt-ended and cloned into the blunt-ended HindIII site of the gateway destination vector pGWB1 carrying kanamycin and hygromycin resistance genes [51], resulting in ProCUC2 pGWB1. To generate entry clones, cDNAs were first amplified with gene-specific primers and then with the attB adaptor primers listed in Supplementary Table S5 and cloned into pDONR221 by BP reaction. The inserts were then transferred to ProCUC2 pGWB1 by LR reaction. For ProCUC2:GUS, the GUS gene fragment was transferred from the entry clone pENTR-gus (Invitrogen [Waltham, MA, USA]) to ProCUC2 pGWB1 via LR reaction. Plant transformation was carried out by the floral dip method [52]. The Pro $2 k b$ and Pro1kb reporter constructs of the KLU gene contain -2066 to +16 and -1061 to +16 sequences from the start codon, respectively, at their $5^{\prime}$ end of vYFPer, an ER-localized version of Venus, and -85 to +338 sequence from the stop codon at their $3^{\prime}$ end and are cloned in the binary vector pBarMAP [53]. Both constructs were transformed to Ler.

\subsection{Rescue Experiments}

Double-homozygous cuc1-5 cuc2-3 plants were seedling lethal and did not produce flowers, thus each construct for the rescue experiments was transformed to cuc1-5 cuc2-3/+ plants via the floral dip method. $\mathrm{T}_{1}$ seeds were selected for drug resistance on Murashige-Skoog plates [54] and the phenotypes of the resistant seedlings were scored. Subsequently, double-homozygous plants were identified by PCR-based genotyping. For analyses of the combined effect of STM with LAS, one transgenic line containing the ProCUC2:STM transgene was maintained and $\mathrm{T}_{2}$ plants with the cuc1-5 cuc2-3/+ genotype were crossed with two independent lines of ProCUC2:LAS with the cuc1-5 cuc2-3/+ genotype. The seedling phenotype was first scored in the $\mathrm{F}_{2}$ generation and the genotype of the CUC2 locus as well as the presence of each transgene was subsequently analyzed. The same ProCUC2:STM line was used for analysis of the combined effect of STM with the rest of the genes and was crossed with two independent transgenic lines for each gene. Scoring of the seedling phenotype and subsequent genotyping were carried out in the $\mathrm{F}_{1}$ generation. To classify the phenotypes into the mild and strong categories, we examined the presence or absence of visible shoot under a binocular. The absence of shoot production was confirmed at 14 dag or later.

\subsection{Histological Analysis}

In situ hybridization was carried out as described previously [17]. As a template for the UFO probe, we used pDW221.1 [55]. For generating templates for other probes, gene-specific fragments were PCR-amplified using the primers listed in Supplementary Table S6 and cloned into PCR ${ }^{\text {TM}}$-Blunt II-TOPO. For GUS detection, embryos were dissected and immediately stained in staining solution with $5 \mathrm{mM}$ ferricyanide and ferrocyanide [56]. Embryos and seedling apices were visualized after clearing as described previously [17]..

\subsection{DEX Induction and $q R T-P C R$}

Induction of CUC1-GR with DEX and expression analysis by qRT-PCR were carried out as described previously [22]. Primers used for qRT-PCR are listed in Supplementary Table S7. 
Supplementary Materials: Supplementary Materials can be found at http://www.mdpi.com/1422-0067/21/16/ 5864/s1. Table S1: List of 52 Genes Downregulated in cuc1 cuc2 Embryos in Microarray Experiments. Table S2: Quantitative RT-PCR of 51 Selected Genes in Heart Stage Embryos. Table S3: Quantitative RT-PCR of 51 Selected Genes in Bending Cotyledon Stage Embryos. Table S4: Mutants used in this analysis. Table S5: Gene-Specific Primers for Rescue Constructs. Table S6: Primers used for cloning probe templates for in situ hybridization. Table S7: Primers used for qRT-PCR experiments. Figure S1: Expression of candidate downstream genes in wild-type Ler and cuc1-1 cuc2-1 embryos. Figure S2: Examples of rescued and non-rescued plants. Text S1: Screening of additional genes regulated by CUC1 and CUC2.

Author Contributions: Conceptualization, M.A. and M.T.; Transcriptome analysis and qRT-PCR verification, Y.T.; In situ hybridization, M.A.; Expression analysis of CUC1-GR, S.S.; Rescue experiments and loss-of-function analysis of CUC downstream genes, H.O., M.K., M.A., S.T., M.R.K. and M.M.; Genetic analysis of KLU and STM, M.A.; Reporter analysis of KLU, R.I., M.A. and M.L.; Original draft preparation, M.A. All authors have read and agreed to the published version of the manuscript.

Funding: This work was supported by the Ministry of Education, Culture, Sports, Science, and Technology of Japan (grant numbers 23012031, 2411400901, 18H04842, 20H04889 to M.A.); the Japan Society for the Promotion of Science (Grant No. 23370023, 16K07401 to M.A.); and Takeda Science Foundation (to M.A.).

Acknowledgments: We thank Detlef Weigel for pDW221.1, Chiyoko Machida for the knat6-2 allele, Ayako Yamaguchi and Arabidopsis Biological Resource Center (ABRC) for the klu alleles, Rüdiger Simon for CLV3::GUS, and RIKEN Biological Resource Center (BRC) for RAFL cDNAs (pda12509 and pda11315). We also thank Mizuki Yamada for critical reading and Edanz (www.edanzediting.co.jp) for editing the English text of a draft of this manuscript. Finally, we thank Etsuko Habe, Seiko Ishihara, Naoko Fujihara, Eriko Tanaka, Maki Niidome, Mie Matsubara, and Kazuko Onga for technical assistance.

Conflicts of Interest: The authors declare no conflict of interest. The funders had no role in the design of the study; in the collection, analyses, or interpretation of data; in the writing of the manuscript, or in the decision to publish the results.

\section{References}

1. Weigel, D.; Jürgens, G. Stem cells that make stems. Nature 2002, 415, 751-754. [CrossRef]

2. Barton, M.K. Twenty years on: The inner workings of the shoot apical meristem, a developmental dynamo. Dev. Biol. 2010, 341, 95-113. [CrossRef]

3. Gaillochet, C.; Lohmann, J.U. The never-ending story: From pluripotency to plant developmental plasticity. Development 2015, 142, 2237-2249. [CrossRef]

4. Aida, M.; Ishida, T.; Fukaki, H.; Fujisawa, H.; Tasaka, M. Genes involved in organ separation in Arabidopsis: An analysis of the cup-shaped cotyledon mutant. Plant Cell 1997, 9, 841-857. [CrossRef]

5. Takada, S.; Hibara, K.; Ishida, T.; Tasaka, M. The CUP-SHAPED COTYLEDON1 gene of Arabidopsis regulates shoot apical meristem formation. Development 2001, 128, 1127-1135.

6. Hibara, K.; Karim, M.R.; Takada, S.; Taoka, K.; Furutani, M.; Aida, M.; Tasaka, M. Arabidopsis CUP-SHAPED COTYLEDON3 regulates postembryonic shoot meristem and organ boundary formation. Plant Cell 2006, 18, 2946-2957. [CrossRef]

7. McConnell, J.R.; Emery, J.; Eshed, Y.; Bao, N.; Bowman, J.; Barton, M.K. Role of Phabulosa and Phavoluta in determining radial patterning in shoots. Nature 2001, 411, 709-713. [CrossRef] [PubMed]

8. Emery, J.F.; Floyd, S.K.; Alvarez, J.; Eshed, Y.; Hawker, N.P.; Izhaki, A.; Baum, S.F.; Bowman, J.L. Radial patterning of Arabidopsis shoots by class III HD-ZIP and KANADI genes. Curr. Biol. 2003, 13, 1768-1774. [CrossRef]

9. Prigge, M.J.; Otsuga, D.; Alonso, J.M.; Ecker, J.R.; Drews, G.N.; Clark, S.E. Class III homeodomain-leucine zipper gene family members have overlapping, antagonistic, and distinct roles in Arabidopsis development. Plant Cell 2005, 17, 61-76. [CrossRef] [PubMed]

10. Zhang, Z.; Tucker, E.; Hermann, M.; Laux, T. A molecular framework for the embryonic initiation of shoot meristem stem cells. Dev. Cell 2017, 40, 264-277.e264. [CrossRef] [PubMed]

11. Knauer, S.; Holt, A.L.; Rubio-Somoza, I.; Tucker, E.J.; Hinze, A.; Pisch, M.; Javelle, M.; Timmermans, M.C.; Tucker, M.R.; Laux, T. A protodermal miR394 signal defines a region of stem cell competence in the Arabidopsis shoot meristem. Dev. Cell 2013, 24, 125-132. [CrossRef] [PubMed] 
12. Aida, M.; Tasaka, M. Genetic control of shoot organ boundaries. Curr. Opin. Plant Biol. 2006, 9, 72-77. [CrossRef] [PubMed]

13. Aida, M.; Tasaka, M. Morphogenesis and patterning at the organ boundaries in the higher plant shoot apex. Plant Mol. Biol. 2006, 60, 915-928. [CrossRef] [PubMed]

14. Takeda, S.; Aida, M. Establishment of the embryonic shoot apical meristem in Arabidopsis thaliana. J. Plant Res. 2011, 124, 211-219. [CrossRef]

15. Rast, M.I.; Simon, R. The meristem-to-organ boundary: More than an extremity of anything. Curr. Opin. Genet. Dev. 2008, 18, 287-294. [CrossRef]

16. Wang, Q.; Hasson, A.; Rossmann, S.; Theres, K. Divide et impera: Boundaries shape the plant body and initiate new meristems. New Phytol. 2016, 209, 485-498. [CrossRef]

17. Aida, M.; Ishida, T.; Tasaka, M. Shoot apical meristem and cotyledon formation during Arabidopsis embryogenesis: Interaction among the CUP-SHAPED COTYLEDON and SHOOT MERISTEMLESS genes. Development 1999, 126, 1563-1570.

18. Raman, S.; Greb, T.; Peaucelle, A.; Blein, T.; Laufs, P.; Theres, K. Interplay of miR164, CUP-SHAPED COTYLEDON genes and LATERAL SUPPRESSOR controls axillary meristem formation in Arabidopsis thaliana. Plant J. 2008, 55, 65-76. [CrossRef]

19. Belles-Boix, E.; Hamant, O.; Witiak, S.M.; Morin, H.; Traas, J.; Pautot, V. KNAT6: An Arabidopsis homeobox gene involved in meristem activity and organ separation. Plant Cell 2006, 18, 1900-1907. [CrossRef]

20. Hibara, K.; Takada, S.; Tasaka, M. CUC1 gene activates the expression of SAM-related genes to induce adventitious shoot formation. Plant J. 2003, 36, 687-696. [CrossRef]

21. Daimon, Y.; Takabe, K.; Tasaka, M. The CUP-SHAPED COTYLEDON genes promote adventitious shoot formation on calli. Plant Cell Physiol. 2003, 44, 113-121. [CrossRef] [PubMed]

22. Takeda, S.; Hanano, K.; Kariya, A.; Shimizu, S.; Zhao, L.; Matsui, M.; Tasaka, M.; Aida, M. CUP-SHAPED COTYLEDON1 transcription factor activates the expression of LSH4 and LSH3, two members of the ALOG gene family, in shoot organ boundary cells. Plant. J. 2011, 66, 1066-1077. [CrossRef] [PubMed]

23. Tian, C.H.; Zhang, X.N.; He, J.; Yu, H.P.; Wang, Y.; Shi, B.H.; Han, Y.Y.; Wang, G.X.; Feng, X.M.; Zhang, C.; et al. An organ boundary-enriched gene regulatory network uncovers regulatory hierarchies underlying axillary meristem initiation. Mol. Syst. Biol. 2014, 10. [CrossRef] [PubMed]

24. Greb, T.; Clarenz, O.; Schäfer, E.; Müller, D.; Herrero, R.; Schmitz, G.; Theres, K. Molecular analysis of the LATERAL SUPPRESSOR gene in Arabidopsis reveals a conserved control mechanism for axillary meristem formation. Genes Dev. 2003, 17, 1175-1187. [CrossRef] [PubMed]

25. Anastasiou, E.; Kenz, S.; Gerstung, M.; MacLean, D.; Timmer, J.; Fleck, C.; Lenhard, M. Control of plant organ size by KLUH/CYP78A5-dependent intercellular signaling. Dev. Cell 2007, 13, 843-856. [CrossRef] [PubMed]

26. Wang, J.W.; Schwab, R.; Czech, B.; Mica, E.; Weigel, D. Dual effects of miR156-targeted SPL genes and CYP78A5/KLUH on plastochron length and organ size in Arabidopsis thaliana. Plant Cell 2008, 20, 1231-1243. [CrossRef]

27. Aoyama, T.; Chua, N.H. A glucocorticoid-mediated transcriptional induction system in transgenic plants. Plant J. 1997, 11, 605-612. [CrossRef]

28. Scofield, S.; Murison, A.; Jones, A.; Fozard, J.; Aida, M.; Band, L.R.; Bennett, M.; Murray, J.A.H. Coordination of meristem and boundary functions by transcription factors in the SHOOT MERISTEMLESS regulatory network. Development 2018, 145. [CrossRef]

29. Takano, S.; Niihama, M.; Smith, H.M.; Tasaka, M.; Aida, M. gorgon, a novel missense mutation in the SHOOT MERISTEMLESS gene, impairs shoot meristem homeostasis in Arabidopsis. Plant Cell Physiol. 2010, 51, 621-634. [CrossRef]

30. Byrne, M.E.; Groover, A.T.; Fontana, J.R.; Martienssen, R.A. Phyllotactic pattern and stem cell fate are determined by the Arabidopsis homeobox gene BELLRINGER. Development 2003, 130, 3941-3950. [CrossRef]

31. Itoh, J.I.; Hasegawa, A.; Kitano, H.; Nagato, Y. A recessive heterochronic mutation, plastochron1, shortens the plastochron and elongates the vegetative phase in rice. Plant Cell 1998, 10, 1511-1522. [CrossRef] [PubMed]

32. Miyoshi, K.; Ahn, B.O.; Kawakatsu, T.; Ito, Y.; Itoh, J.I.; Nagato, Y.; Kurata, N. PLASTOCHRON1, a timekeeper of leaf initiation in rice, encodes cytochrome P450. Proc. Natl. Acad. Sci. USA 2004, 101, 875-880. [CrossRef] [PubMed] 
33. Brand, U.; Grünewald, M.; Hobe, M.; Simon, R. Regulation of CLV3 expression by two homeobox genes in Arabidopsis. Plant Physiol. 2002, 129, 565-575. [CrossRef] [PubMed]

34. Long, J.A.; Moan, E.I.; Medford, J.I.; Barton, M.K. A member of the KNOTTED class of homeodomain proteins encoded by the STM gene of Arabidopsis. Nature 1996, 379, 66-69. [CrossRef]

35. Jasinski, S.; Piazza, P.; Craft, J.; Hay, A.; Woolley, L.; Rieu, I.; Phillips, A.; Hedden, P.; Tsiantis, M. KNOX action in Arabidopsis is mediated by coordinate regulation of cytokinin and gibberellin activities. Curr. Biol. 2005, 15, 1560-1565. [CrossRef]

36. Yanai, O.; Shani, E.; Dolezal, K.; Tarkowski, P.; Sablowski, R.; Sandberg, G.; Samach, A.; Ori, N. Arabidopsis KNOXI proteins activate cytokinin biosynthesis. Curr. Biol. 2005, 15, 1566-1571. [CrossRef]

37. Scofield, S.; Dewitte, W.; Nieuwland, J.; Murray, J.A.H. The Arabidopsis homeobox gene SHOOT MERISTEMLESS has cellular and meristem-organisational roles with differential requirements for cytokinin and CYCD3 activity. Plant J. 2013, 75, 53-66. [CrossRef]

38. Kierzkowski, D.; Runions, A.; Vuolo, F.; Strauss, S.; Lymbouridou, R.; Routier-Kierzkowska, A.L.; Wilson-Sánchez, D.; Jenke, H.; Galinha, C.; Mosca, G.; et al. A growth-based framework for leaf shape development and diversity. Cell 2019, 177, 1405-1418.e1417. [CrossRef]

39. Hake, S.; Smith, H.M.; Holtan, H.; Magnani, E.; Mele, G.; Ramirez, J. The role of knox genes in plant development. Annu. Rev. Cell Dev. Biol. 2004, 20, 125-151. [CrossRef]

40. Bhatt, A.M.; Etchells, J.P.; Canales, C.; Lagodienko, A.; Dickinson, H. VAAMANA-A BEL1-like homeodomain protein, interacts with KNOX proteins BP and STM and regulates inflorescence stem growth in Arabidopsis. Gene 2004, 328, 103-111. [CrossRef]

41. Cole, M.; Nolte, C.; Werr, W. Nuclear import of the transcription factor SHOOT MERISTEMLESS depends on heterodimerization with BLH proteins expressed in discrete sub-domains of the shoot apical meristem of Arabidopsis thaliana. Nucleic Acids Res. 2006, 34, 1281-1292. [CrossRef] [PubMed]

42. Rutjens, B.; Bao, D.; van Eck-Stouten, E.; Brand, M.; Smeekens, S.; Proveniers, M. Shoot apical meristem function in Arabidopsis requires the combined activities of three BEL1-like homeodomain proteins. Plant J. 2009, 58, 641-654. [CrossRef] [PubMed]

43. Cao, X.; Wang, J.; Xiong, Y.; Yang, H.; Yang, M.; Ye, P.; Bencivenga, S.; Sablowski, R.; Jiao, Y. A self-activation loop maintains meristematic cell fate for branching. Curr. Biol. 2020. [CrossRef] [PubMed]

44. Tucker, D.J. Endogenous growth regulators in relation to side shoot development in the tomato. New Phytol. 1976, 77, 561-568. [CrossRef]

45. Schumacher, K.; Schmitt, T.; Rossberg, M.; Schmitz, G.; Theres, K. The Lateral suppressor (Ls) gene of tomato encodes a new member of the VHIID protein family. Proc. Natl. Acad. Sci. USA 1999, 96, 290-295. [CrossRef]

46. Zhang, Q.Q.; Wang, J.G.; Wang, L.Y.; Wang, J.F.; Wang, Q.; Yu, P.; Bai, M.Y.; Fan, M. Gibberellin repression of axillary bud formation in Arabidopsis by modulation of DELLA-SPL9 complex activity. J. Integr. Plant Biol. 2020, 62, 421-432. [CrossRef]

47. Somssich, M.; Je, B.I.; Simon, R.; Jackson, D. CLAVATA-WUSCHEL signaling in the shoot meristem. Development 2016, 143, 3238-3248. [CrossRef]

48. Smith, H.M.; Hake, S. The interaction of two homeobox genes, BREVIPEDICELLUS and PENNYWISE, regulates internode patterning in the Arabidopsis inflorescence. Plant Cell 2003, 15, 1717-1727. [CrossRef]

49. Sabatini, S.; Heidstra, R.; Wildwater, M.; Scheres, B. SCARECROW is involved in positioning the stem cell niche in the Arabidopsis root meristem. Genes Dev. 2003, 17, 354-358. [CrossRef]

50. Taoka, K.; Yanagimoto, Y.; Daimon, Y.; Hibara, K.; Aida, M.; Tasaka, M. The NAC domain mediates functional specificity of CUP-SHAPED COTYLEDON proteins. Plant J. 2004, 40, 462-473. [CrossRef]

51. Nakagawa, T.; Kurose, T.; Hino, T.; Tanaka, K.; Kawamukai, M.; Niwa, Y.; Toyooka, K.; Matsuoka, K.; Jinbo, T.; Kimura, T. Development of series of gateway binary vectors, pGWBs, for realizing efficient construction of fusion genes for plant transformation. J. Biosci. Bioeng. 2007, 104, 34-41. [CrossRef] [PubMed]

52. Clough, S.J.; Bent, A.F. Floral dip: A simplified method for Agrobacterium-mediated transformation of Arabidopsis thaliana. Plant J. 1998, 16, 735-743. [CrossRef] [PubMed]

53. Adamski, N.M.; Anastasiou, E.; Eriksson, S.; O'Neill, C.M.; Lenhard, M. Local maternal control of seed size by KLUH/CYP78A5-dependent growth signaling. Proc. Natl. Acad. Sci. USA 2009, 106, 20115-20120. [CrossRef] [PubMed] 
54. Fukaki, H.; Fujisawa, H.; Tasaka, M. SGR1, SGR2, SGR3: Novel genetic loci involved in shoot gravitropism in Arabidopsis thaliana. Plant. Physiol. 1996, 110, 945-955. [CrossRef] [PubMed]

55. Lee, I.; Wolfe, D.S.; Nilsson, O.; Weigel, D. A LEAFY co-regulator encoded by UNUSUAL FLORAL ORGANS. Curr. Biol. 1997, 7, 95-104. [CrossRef]

56. Sessions, A.; Weigel, D.; Yanofsky, M.F. The Arabidopsis thaliana MERISTEM LAYER 1 promoter specifies epidermal expression in meristems and young primordia. Plant J. 1999, 20, 259-263. [CrossRef]

(C) 2020 by the authors. Licensee MDPI, Basel, Switzerland. This article is an open access article distributed under the terms and conditions of the Creative Commons Attribution (CC BY) license (http://creativecommons.org/licenses/by/4.0/). 